\title{
TIME SERIES ANALYSIS OF INFECTIOUS DISEASE MORTALITY IN UKRAINE (1965-2015)
}

\author{
Hennadii Mokhort, Olga Sokolovska \\ Epidemiology, Bogomolets National Medical University, Kyiv, Ukraine \\ Objective
}

The aim of our work is to determine the main trends and structure in infectious disease mortality in Ukraine over the last 50 years.

\section{Introduction}

Monitoring of long-term infectious disease mortality trends is of great value to national public health systems both in estimation of the efficacy of preventive programs, and in development of the new strategies of preventive measures. In the developed countries, there are a number of studies with long-term time series of infectious disease mortality analysis in epidemiological and historical aspects. Our research was based on the work by Armstrong GL, Conn LA and Pinner RW, 1999. Literature review revealed that such analysis has been never carried out in Ukraine up to now.

\section{Methods}

Our study is designed as a descriptive retrospective epidemiological analysis. We constructed time series of infectious mortality in all oblasts of Ukraine during the period of 1965-2015 years. We used annual statistical forms C-8 "Distribution of deceased by sex, age and death cause" provided by Ministry of Health. The cause of death was accounted in accordance with international statistical classification of diseases, injuries, and causes of death: based on the recommendations of the ninth revision conference, 1975 . We analyzed infectious diseases belonging to the class of Infectious and parasitic diseases (45 nosology and nosology groups - codes 001 - 139). We also included into our analysis some other infectious diseases belonging to other classes: Neoplasm (cervix carcinoma - code 180); Heart diseases (Rheumatic fever, rheumatic heart disease - codes 390-398); Diseases of the respiratory system (Acute Respiratory Infection, influenza, viral pneumonia, pneumococcal pneumonia, other acute forms of pneumonia codes 460-466, 487, 480, 481, 482, 483, 485, 486); diseases of nervous system (non-infectious and non-parasitic meningitis, codes 320-322).

Therefore, all infections that are reported in Ukraine were included to this research. Nosologies were grouped using several disease classifications: in accordance with International classification (belonging or not to Infectious disease class); by transmission method or localization of an infectious agent (respiratory, intestinal or alimentary, blood borne, contact and other infections); by ecological principle (anthroponosis, zoonosis, sapronosis and other).

All time series were divided onto two periods: 1965-1991 (soviet period) and 1992-2015 (period of independent Ukraine). Average mortality (mortality coefficient) of these periods was compared to each other for calculation of percentage decrease/increase of each disease's mortality rate. Additionally, we determined the proportion (\%) of infectious mortality compare to the total mortality of population of Ukraine. Limited scope of this study does not allow us to present data regarding the age distribution, thus we focus on general characteristics. Although the practice of presenting data was changed over the course of 50 years covered by this abstract, the data are comparable and can be used for analysis.

\section{Results}

Total number of fatal cases caused by infectious diseases in Ukraine during 1965-2015 years is 1,268,560 or 4.05\% of all deaths caused by different reasons. 550,329 deaths or $43.38 \%$ of all infectious deaths belong to class of infectious and parasitic diseases, other 718,231 or $56.62 \%$ belong to infectious diseases of other classes. Percentage of respiratory infections is $80.28 \%$, intestinal infections $-1.72 \%$, blood infections $-16.94 \%$ and other infections $-1.05 \%$. Additionally, proportion of anthroponosis is $98.31 \%$, proportion of zoonosis $-0.42 \%$, sapronosis $-0.22 \%$, other $-1.05 \%$. During $1965-2015$, percentage of infectious diseases in overall structure was within the range from $10.53 \%$ (1965) to $2.99 \%$ (2015). Overall mortality rate of infectious diseases decreased from 80.49 per 100,000 population (1965) to 41.77 per 100,000 (2015). This finding demonstrates to reduction of overall infectious mortality in Ukraine. It is important to mention that decrease of overall infectious mortality happened simultaneously with an increase of mortality caused by non-infectious diseases. Non-infectious mortality increased from 683.92 per 100000 population (1965) to 1354.77 per 100000 population (2015).

SDS Annual Conference Proceedings 2019. This is an Open Access article distributed under the terms of the Creative Commons AttributionNoncommercial 4.0 Unported License (http://creativecommons.org/licenses/by-nc/3.0/), permitting all non-commercial use, distribution, and reproduction in any medium, provided the original work is properly cited. 
The first 10 causes of death from infectious diseases in Ukraine in 1965-2015 included the following nosological units and infectious groups: 1 . Respiratory tuberculosis and other forms of tuberculosis (30.3\%); 2 . Acute respiratory infections + Influenza + Viral pneumonia + Pneumococcal pneumonia and Other acute pneumonias $(28.36 \%)$; 3. Acute rheumatic fever + Chronic rheumatic heart disease (15.93\%); 4. Malignant neoplasm of cervix uteri (10.42\%); 5. AIDS (4.8\%); 6. Septicemia (2.86\%); 7. Meningococcal infection + Meningitis, excluding infectious and parasitic meningitis (2.64\%); 8. Other infectious and parasitic diseases and long-term effects of other infectious and parasitic diseases (1.05\%); 9. Acute intestinal infections due to unspecified micro-organisms and ill-defined, including toxical dyspepsia $(0.93 \%)$ and 10 . Viral hepatitis $(0.79 \%)$.

The average mortality rate declined for the most infectious diseases during 1992-2015 comparing to 1965-1991. For certain diseases or their groups, the range varied from $12.71 \%$ (pneumonia) to $92.69 \%$ (influenza). The biggest decrease was observed in intestinal infections group (up to $73.99 \%$ ). All respiratory infections demonstrated a decrease to $18.14 \%$; other infections with unknown path of transmission resulted in $4.6 \%$ decrease, but blood borne infections demonstrated an increase of 59.72\% (mostly caused by AIDS). Some other infections also demonstrated increase in mortality rate: foodborne botulism - up to $29.94 \%$, tuberculosis

- up to $26.52 \%$, diphtheria - up to $376,53 \%$, erysipelas - to $371,26 \%$, AIDS - to $100 \%$.

\section{Conclusions}

Infectious diseases are not the main mortality cause among the population of Ukraine during the past 50 years. Over the last halfcentury, the proportion of infectious diseases in the mortality structure of the population of Ukraine demonstrated a decreasing tendency, while non-infectious disease mortality had an opposite trend, which can be explained by epidemiological transition (Omran AR, 1971). However, there is always a possibility of rapid spreading of infectious diseases and increasing their proportion in the structure of total mortality. Possible growth of mortality rate caused by AIDS, tuberculosis and diphtheria is an issue of concern. International experience demonstrated that these three infections could be successfully controlled. The long-term trends of AIDS, tuberculosis and diphtheria mortality rates in Ukraine require regulatory interventions and show the need for emergency measures by the state services to these and some other infections, including vaccine-controlled. Thus, our study of the long-term trends of infectious mortality can be used to make decisions of public health in Ukraine on the control of infectious morbidity and mortality.

\section{Acknowledgement}

The author is thankful to the Ministry of Health of Ukraine for giving access and sharing their invaluable data set on the disease mortality.

\section{References}

Armstrong GL, Conn LA, Pinner RW. 1999. Trends in Infectious Disease Mortality in the United States During the $20^{\text {th }}$ Century. JAMA. 281(1), 61-66. PubMed https://doi.org/10.1001/jama.281.1.61

Omran AR. 1971. The epidemiologic transition: a theory of the epidemiology of population change. Milbank Fund Q. 49, 509-38. PubMed https://doi.org/10.2307/3349375

ISDS Annual Conference Proceedings 2019. This is an Open Access article distributed under the terms of the Creative Commons AttributionNoncommercial 4.0 Unported License (http://creativecommons.org/licenses/by-nc/3.0/), permitting all non-commercial use, distribution, and reproduction in any medium, provided the original work is properly cited. 